\title{
VEGETATION COVERAGE AND IMPERVIOUS SURFACE AREA ESTIMATED BASED ON THE ESTARFM MODEL AND REMOTE SENSING MONITORING
}

\author{
Hu Rongming ${ }^{1}$, Wang Shu ${ }^{1}$, Guo Jiao ${ }^{2}$, Guo Liankun ${ }^{1}$ \\ ${ }^{1}$ College of Geomatics, Xi'an University of Science and Technology, China, wshu3928@163.com \\ ${ }^{2} \mathrm{Xi}$ ' an Institute of Prospecting and Mapping, China, 444313346@qq.com
}

KEY WORDS: ESTARFM model, vegetation coverage, impervious surface area, spatial differentiation, correlation analysis

\begin{abstract}
:
Impervious surface area and vegetation coverage are important biophysical indicators of urban surface features which can be derived from medium-resolution images. However, remote sensing data obtained by a single sensor are easily affected by many factors such as weather conditions, and the spatial and temporal resolution can not meet the needs for soil erosion estimation. Therefore, the integrated multi-source remote sensing data are needed to carry out high spatio-temporal resolution vegetation coverage estimation. Two spatial and temporal vegetation coverage data and impervious data were obtained from MODIS and Landsat 8 remote sensing images. Based on the Enhanced Spatial and Temporal Adaptive Reflectance Fusion Model (ESTARFM), the vegetation coverage data of two scales were fused and the data of vegetation coverage fusion (ESTARFM FVC) and impervious layer with high spatiotemporal resolution (30m, 8day) were obtained. On this basis, the spatial variability of the seepage-free surface and the vegetation cover landscape in the study area was measured by means of statistics and spatial autocorrelation analysis. The results showed that: 1) ESTARFM FVC and impermeable surface have higher accuracy and can characterize the characteristics of the biophysical components covered by the earth's surface; 2) The average impervious surface proportion and the spatial configuration of each area are different, which are affected by natural conditions and urbanization. In the urban area of Xi'an, which has typical characteristics of spontaneous urbanization, landscapes are fragmented and have less spatial dependence.
\end{abstract}

\section{INTRODUCTION}

Impervious surface area and vegetation cover are important biophysical indexes used to characterize the urban surface features. They can be used for the quantitative description of urbanization degree, evaluation of ecological environment effect, construction of ecological environment model. The impervious surface ratio refers to the proportion of the area occupied by impervious surfaces such as asphalt and cement per unit area, representing the degree of human activities(Chester L,1996a). Vegetation coverage can be defined as the vertical projected area of vegetation per unit area, characterizing the natural ecological environment(Gitelson A A,2002a). The application of impervious surfaces and vegetation coverage to landscape pattern analysis can not only characterize the land cover characteristics but also characterize the differences in the biophysical composition of the same land use types.

Although remote sensing technology has acquired a large amount of remote sensing data, due to the restriction of technical conditions, researchers must choose the time-space scale when using remote sensing data for terrestrial ecosystem monitoring (Price J C,1994a). At present, the resolution of remote sensing data can be roughly divided into two types: one is the data with high spatial resolution and low temporal resolution, such as TM, ETM +, ASTER, etc. These types of data have high recognition accuracy and long revisit period. Cloud weather images are difficult to obtain, there are some problems such as discontinuity of dynamic monitoring of urban surface features, and the other is low spatial temporal resolution, such as AVHRR and MODIS, which have better timeliness, but lower spatial resolution leads to primitive image quality is not ideal, making the urban surface feature estimation data subject to high uncertainty and eventually, impacting many applications evaluation results. Therefore, using a single means to obtain remote sensing data to estimate the vegetation coverage in a certain area can easily lead to an unsatifacory spatiotemporal resolution; if the quality of the original remote sensing image is not ideal, the estimation data will be greatly uncertain, and affect other results of application evaluations.

Therefore, the fusion of different scales of remote sensing data to estimate the urban surface features will help to improve the current spatial and temporal resolution of data, the status of the weak surface dynamic monitoring. In this paper, Xi'an, the capital city of Shaanxi province, China,was chosen as the study area. Based on the ESTARFM model, the vegetation cover and impervious layer were estimated based on remote sensing data. Then the temporal and spatial variation of vegetation coverage in Xi'an area were analyzed. The spatial differentiation measure was carry out as a supplement to the traditional landscape pattern analysis based on landscape types.

\section{STUDY AREA AND DATA PREPROCESSING}

\subsection{Study area and data source}

Xi'an is located in the Guanzhong Basin in the middle of the Weihe River basin, covering the area of $10108 \mathrm{~km}^{2}$ with longitude and latitude ranging from 107.40E to $109.49 \mathrm{E}$ and $33.42 \mathrm{~N}$ to $34.45 \mathrm{~N}$ respectively. The east-west length is about $204 \mathrm{~km}$ and the north-south width is $116 \mathrm{~km}$, including ten districts (Chang'an, Baqiao, Yanta, Xincheng, Lianhu, Weiyang, Yanliang, Lintong, Gaoling and Huyi ) and two counties (Lantian and Zhouzhi)). The overall topography of Xi'an is characterized by a large difference in elevation between the north and south and a clear landscape of East and West plains and tableland. Among them, Li Shan is the hilly region in the east, the Qinling Mountains in the south and the flood plain in the Weihe river basin in the west and north. 
The data used in this paper include: Landsat8, MODIS09A1. The data list is shown in Table 1.

\begin{tabular}{ccccc}
\hline Data & Get Time & $\begin{array}{c}\text { Spatial } \\
\text { resolution } \\
(\mathrm{m})\end{array}$ & $\begin{array}{c}\text { Time } \\
\text { resolution } \\
(\text { day })\end{array}$ & Attributes \\
\hline $\begin{array}{c}\text { Landsat } \\
\text { 8 OLI }\end{array}$ & $\begin{array}{c}2014 \\
\text { cloudless } \\
\text { data }\end{array}$ & 30 & 16 & DN value \\
MODIS & $\begin{array}{c}2014 \\
\text { cloudless } \\
\text { 09A } 1\end{array}$ & 500 & 8 & $\begin{array}{c}\text { Surface } \\
\text { reflectivity }\end{array}$ \\
\hline
\end{tabular}

Table 1.The list of data used in the study

\subsection{Data preprocessing}

2.2.1 Landsat 8 data preprocessing: Landsat8 data was downloaded from the USGS GLOVIS (http://glovis.usgs.gov/) website and the map projection is UTM-WGS84 projection coordinate system for correction of landsat8 images and atmospheric correction to eliminate various aberrations in radiance, including Sensors detect differences in system performance, atmospheric, solar elevation, and terrain-related errors, and convert the grayscale values of ground targets to actual surface reflectance values. Landsat OLI satellite data preprocessing process is shown in Figure 1.

2.2.2 MOD09A1 data preprocessing: MODIS09A1 is a terrestrial Level 3 standard product that uses MODIS Reprojection Tool (MRT) to preprocess MOD09A1 data. The MRT tool was used to convert the HDF format to the GEOTIFF format, transform the SIN map projection into a WGS / UTM1984 49N projection, and select the Nearest Neighbor to stitch and resample the image for the high temporal fusion data input of the ESTARFM model Ready. Uses UTM projection vector files to crop the stitched image.

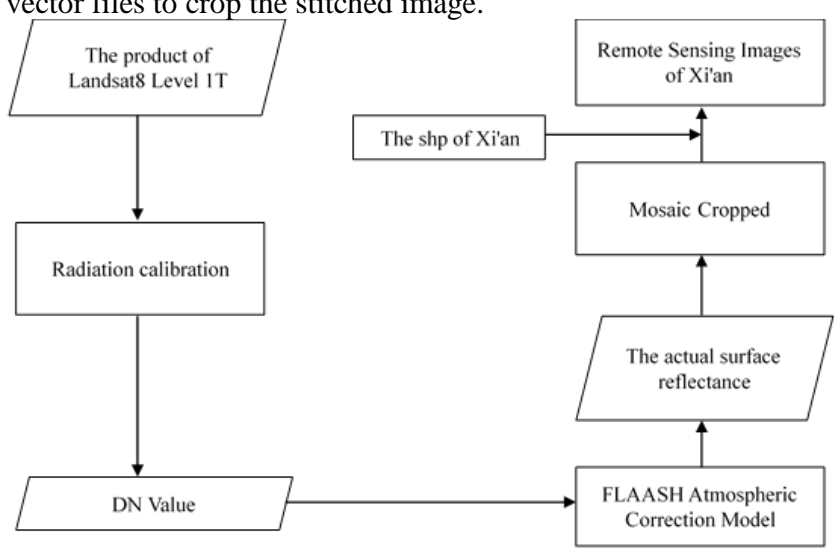

Figure 1. Landsat8 data pre-processing flow chart

\section{METHODS}

\subsection{Impermeable layer extraction}

The methods for estimating the distribution of impervious layers of remote sensing data are mainly divided into the following categories(Li,2016): (1) The impermeable layer extraction of medium- and low-resolution remote sensing data mainly includes spectral mixed analysis(Ridd M K,1995), regression method ( $\mathrm{Lu}$ D,2014), classification method (Hu X F,2009;Zhang Y,2014), index method(Chong Liu,2013); (2) Urban impermeable layer extraction of high-resolution remote sensing data mainly includes pixel-based method (Goetz S J,2003a) object-oriented method (Moran E F,2010), multivariate data fusion method (Mohapatra R P,2010).

3.1.1 V-H-L-S mode: Based on the classical V-I-S mode, $\mathrm{Wu}(\mathrm{Wu} \mathrm{C}$, Murray A T,2003) improved it by subdividing impermeable layers into high-reflectivity and low-reflectivity features. The processing flow is shown in Figure2.

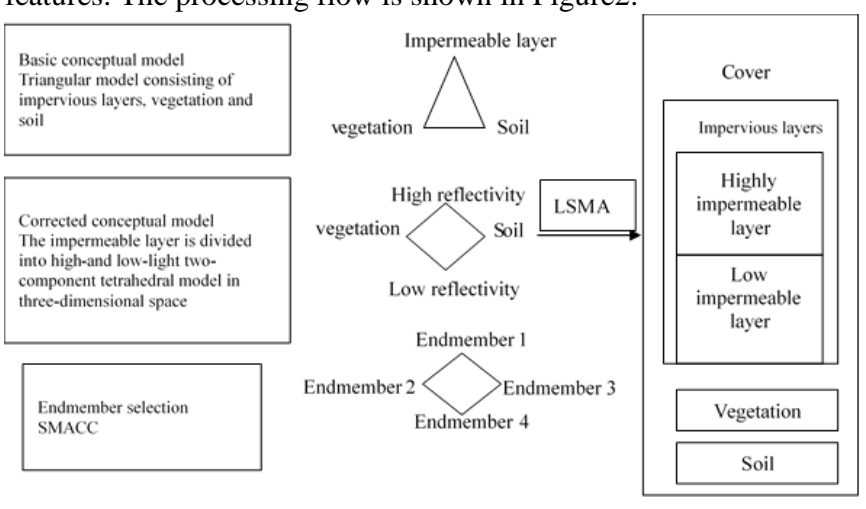

Figure 2. V-H-L-S processing flow chart

3.1.2 Endmember Selection: After converting the pixel's DN value to a standard reflectance, the endmember spectra and abundance images are extracted from the image using a Sequential Maximum Angle Convex Cone (SMACC). It provides a faster, more automated way to get the endmember spectrum. The SMACC method is based on the convex cone model (also known as residual minimization) to identify image endmember spectra with constraints. The pole is used to determine the convex cone, and then the first endmember spectrum is defined; then, an oblique projection with constraints is applied to the existing cone to generate the next endmember spectrum; and the cone is continuously added to generate a new endmember spectrum. This process is repeated until the resulting convex cone includes the existing end-cells (to a certain tolerance) or until the specified number of end-cell spectral classes is satisfied.

3.1.3 Linear Spectral Mixed Decomposition Mode: The reflectivity obtained from remote sensing images is stored in pixels, and the size of the pixels depends on the spatial resolution of the sensor. At a certain resolution, if it is determined that a feature recorded in a pixel is pure, then the pixel is called a pure pixel whose reflectivity is completely determined by the feature. However, due to the high heterogeneity of the Earth's surface, especially the urban surface, a variety of reflections with different reflection spectra in the same pixel often appear, and the reflectivity of the pixel is reflected by various features in the pixel Certain combinations of rates determine that such a pixel is called a "mixed pixel." Spectral hybrid analysis has been widely used as a classical method of impermeable layer extraction based on remote sensing images.

Linear Spectral Mixed Analysis (LSMA) is an effective method to quantify the urban surface coverage components at the subpixel scale. Spectral hybrid analysis assumes that in low- and medium-resolution remote sensing images, each pixel is composed of several features that are mixed at different scales. Spectral mixing analysis was employed to extract the pure spectrum of each feature, that is, the endmember spectrum, and calculate the abundance distribution of each feature. Linear mixed model is the most commonly used mixed model: 


$$
y=\sum_{i=1}^{m} \alpha_{i} \mu_{i}+\varepsilon
$$

Where $y$ is the observed spectral vector of the mixed pixel; $\mathrm{m}$ is the total number of the endpoints; $\mu_{i}$ is the spectral vector of the ith endmember, $\alpha_{i}$ is the abundance ratio of the end $\mathrm{i}$ in a single cell; $\varepsilon$ is the residual difference. $\alpha_{i}$ needs to satisfy $\sum_{i=1}^{m} \alpha_{i}=1$ and $0 \leq \alpha_{i} \leq 1$.

\subsection{Vegetation coverage extraction}

There are many ways of estimating the vegetation coverage based on remote sensing data. The most typical and most widely used vegetation coverage estimation model - pixel binary model is used to inverse the vegetation coverage in the study area.

3.2.1 The concept of pixel dichotomy mode:Pixel dichotomy model (PDM) is a relatively mature method for the estimation of vegetation cover. Remote sensing images are composed of many pixels, each pixel contains two kinds of information, one is vegetation information, the other is soil information. In the same way, the spectral information $S$ observed by the sensor consists of two parts, one is vegetation contribution information $S_{v}$ and the other is soil contribution information $\mathrm{S}_{\mathrm{s}}$, which is composed of two parts of linear weighting $\mathrm{S}$, which can be expressed as:

$$
S=S_{v}+S_{s}
$$

In each mixed pixel, the proportion of the area occupied by vegetation is called the vegetation coverage $f_{c}$ of the pixel, and the proportion of soil is $1-f_{c}$. Assuming that the pixel information of pure vegetation in remote sensing images is $\mathrm{s}_{\text {veg }}$ and the information of bare soil pixels without vegetation cover is $S_{\text {soil }}$, the contributions of vegetation pixels to a mixed pixel can be expressed as:

$$
S_{v}=S_{v e g} * f_{c}
$$

The contribution of soil to pixel is expressed as:

$$
S_{s}=S_{\text {soil }} *\left(1-f_{c}\right)
$$

Therefore, the information $\mathrm{S}$ observed by the remote sensing method is denoted as:

$$
S=S_{v e g} * f_{c}+S_{\text {soil }} *\left(1-f_{c}\right)
$$

In summary:

$$
f_{c}=\frac{S-S_{\text {soil }}}{S_{\text {veg }}-S_{\text {soil }}}
$$

3.2.2 Estimating Vegetation Coverage Using NDVI:NDVI is a parameter that is used to express vegetation coverage. Pixel dichotomy estimates the vegetation coverage using more NDVI values. NDVI is calculated as:

$$
\mathrm{NDVI}=\frac{(N I R-R E D)}{(N I R+R E D)}
$$

Where: NIR represents the reflectivity of the feature in the near infrared band and RED represents the reflectivity of the feature in the infrared band.

The spectral information $\mathrm{S}$ at this time is expressed as follows:

$$
S=N D V I_{\text {veg }} * f_{c}+N D V I \text { soil } *\left(1-f_{c}\right)
$$

In other words, the linear weighted summation of vegetationcovered NDVI and vegetation-free NDVI compose the NDVI of each pixel information, where the weight of vegetation-covered NDVI in this pixel is $f_{c}$, which is vegetation coverage, NDVI weight without vegetation cover is $1-f_{c}$. NDVI veg represents the NDVI value of all vegetation pixels, and $N D V s_{\text {soil }}$ is the NDVI value of all bare soil pixels.

$$
f_{c}=\frac{N D V I-N D V I_{\text {soil }}}{N D V I_{\text {veg }}-N D V I_{\text {soil }}}
$$

$N D V V_{\text {soil }}$ is close to 0 in theory, but is affected by the objective conditions such as soil moisture, type and roughness, $N D V{ }_{\text {soil }}$ is indefinite, that is, its value changes with time and space. Similarly, the theoretical value of $N D V I$ veg is close to 1, but in fact, $N D V{ }_{\text {veg }}$ will also change under the influence of vegetation growth and neighboring pixels in different periods. Therefore, values of $N D V{ }_{\text {soil }}$ and $N D V I_{\text {veg }}$ were determined according to experience.

In this paper, a simple method for determining $N D V t_{v e g}$ and $N D V I_{\text {soil }}$ is adopted, that is, the confidence intervals of vegetation coverage are selected through statistical analysis to determine the values of $N D V I_{\text {veg }}$ and $N D V{ }_{\text {soil }}$. The determination of the confidence interval is based on the cumulative percentage of the total number of pixels occupying thfe pixel corresponding to the NDVI. The corresponding maximum of the cumulative percentage of the interval corresponds to $N D V I_{v e g}$, and the minimum cumulative percentage of the interval corresponds to $N D V I_{\text {soil }}$. In this paper, the NDVI value corresponding to $5 \%$ of the cumulative percentage is the NDVI value corresponding to $95 \%$ of the cumulative percentage, and the vegetation coverage is calculated as:

$$
f_{c}=\frac{N D V-N D V I_{\min }}{N D V I_{\max }-N D V I_{\min }}
$$

Based on this, the flow chart of vegetation coverage calculation using pixel-dichotomy NDVI is shown in Figure 3. 


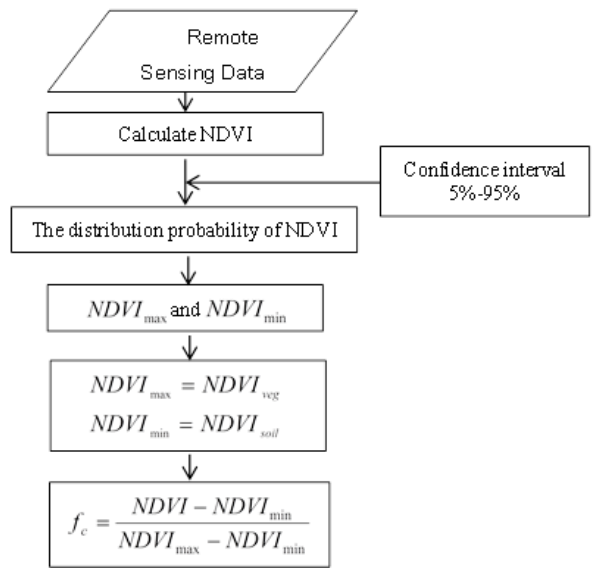

Figure 3. Pixel dichotomy calculation flow chart

In this paper, we use ESTARFM (Enhanced Spatial and Temporal Adaptive Reflectance Fusion Mode) proposed by Zhu(Zhu X, Chen J, Gao F, et al,2010) to enhance the adaptive space-time fusion of remote sensing images. The fusion method can predict the target pixel, taking full account of the adjacent pixels and the target pixel three aspects: First, the geographical distance, the second is the spectral difference, the third is the time difference. The ESTARFM model inputs the high-spatial and high-temporal resolution data of the two periods one after the other and inputs the high-resolution temporal resolution data of the target period to generate the high-spatial-resolution data of the target period. Substituting this data into the model, we first determine the size of the window, search for the pixels similar to the impermeable layer of the center cell in the two phases of Landsat8 impermeable layer data according to the spectral correlation, and then combine the MODIS impermeable layer The weights and conversion coefficients are given to each similar pixel, and then the time weights are calculated according to the MODIS data of the above two phases and the target period. Finally, the center pixels are calculated according to various weights of similar pixels. The specific model steps are shown in the figure4.

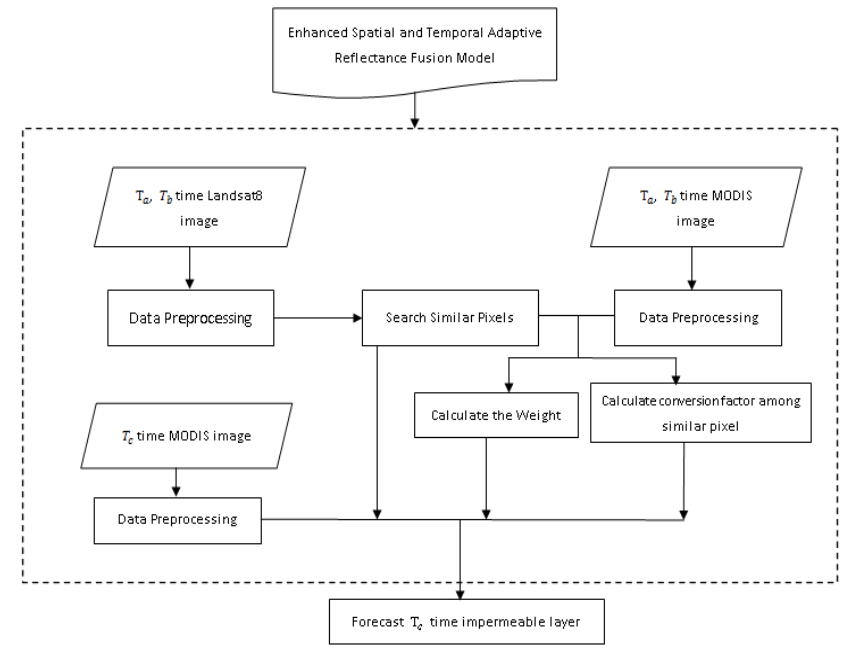

Figure 4. ESTARFM flow chart

In essence, the ESTARFM model moves the entire image one by one by a window size $\mathrm{w}$, thereby determining the prediction value of the pixel at the center of the movement one by one. The prediction value of the center pixel is expressed as (3.11)

$\hat{R}_{\text {high }}\left(x_{w / 2}, y_{w / 2}, T_{b}\right)=T_{a}^{\prime} \hat{R}_{a_{-} \text {high }}\left(x_{w / 2}, y_{w / 2}, T_{b}\right)+T_{c}^{\prime} \hat{R}_{c_{-} \text {high }}\left(x_{w / 2}, y_{w / 2}, T_{b}\right)$
Where $\hat{R}_{\text {righ }}\left(x_{w / 2}, y_{w / 2}, T_{b}\right)$ is the final high-resolution fusion data for the prediction period $T_{b}$, and $\hat{R}_{a_{-}}$high $\left(x_{w / 2}, y_{w / 2}, T_{b}\right)$ and $\hat{R}_{c_{-} h i g h}\left(x_{w / z}, y_{w / 2}, T_{b}\right)$ are the high-resolution data for the original and forecast period $T_{b}$ in $T_{a}$ and $T_{c}$ respectively, which is obtained by (3.12); $T_{a}^{\prime}$ and $T_{c}^{\prime}$ are the time weight, Calculated by (3.13).

$$
\begin{aligned}
& \hat{\mathrm{R}}_{a_{-} \text {high }}\left(x_{w / 2}, y_{w / 2}, T_{b}\right)=R_{\text {high }}\left(x_{w / 2}, y_{w / 2}, T_{t}\right)+ \\
& \sum_{i=1}^{N} \omega_{i} \rho_{i}\left(R_{\text {low }}\left(x_{i}, y_{i}, T_{b}\right)-R_{\text {low }}\left(x_{i}, y_{i}, T_{t}\right)\right),(T=a, c) \\
& T_{t}^{\prime}=\frac{1 /\left|\sum_{i=1}^{w} \sum_{j=1}^{w} R_{\text {low }}\left(x_{i}, y_{i}, T_{t}\right)-\sum_{i=1}^{w} \sum_{j=1}^{w} R_{\text {low }}\left(x_{i}, y_{i}, T_{b}\right)\right|}{\sum_{t=a, c}\left(1 /\left|\sum_{i=1}^{w} \sum_{i=1}^{w} R_{\text {low }}\left(x_{i}, y_{i}, T_{t}\right)-\sum_{i=1}^{w} \sum_{i=1}^{w} R_{\text {low }}\left(x_{i}, y_{i}, T_{b}\right)\right|\right.}
\end{aligned}
$$

Where: $\left(x_{w / 2}, y_{w / 2}\right)$ is the search window center position, which is to be predicted center pixel location, $w$ is the size of the mobile window. $\mathrm{N}$ is the number of similar pixels, $\rho_{i}$ is the conversion factor, and $\omega_{i}$ is the distance weight. $R_{\text {high }}\left(x_{w / 2}, y_{w / 2}, T_{t}\right),(t=a, c)$ is the low temporal resolution data of the original high space in the $T_{a}$ and $T_{c}$ periods. This paper is the Landsat8 impermeable layer data. $R_{\text {low }}\left(x_{i} \cdot y_{i}, T_{b}\right)$ and $R_{\text {low }}\left(x_{i} \cdot y_{i}, T_{\mathrm{t}}\right)$ are the low spatial resolution data of the original high time in the $T_{b}$ and $T_{c}(t=a, c)$ periods respectively. This is MODIS impermeable layer data.

\section{RESULTS AND URBAN IMPERMEABLE LAYER ANALYSIS}

The results of the integration of the 225th day in 2014(20140815)are compared and the coverage of the surface area in Xi'an was analyzed.

\subsection{Qualitative analysis}

Through the visual interpretation, compared with 500m MODIS impermeable layer data, ESTARFM impermeable layer data well preserves the spatial distribution details of Landsat8 impermeable layer data at $30 \mathrm{~m}$ resolution, such as the image texture structure. It can be seen that the ESTARFM FVC data generated by ESTARFM effectively combines the advantages of the two data, increasing both the spatial difference of Landsat8 impermeable layer (and vegetation coverage)and the temporal change of MODIS impermeable layer(and vegetation coverage). The result is shown in figure5. 


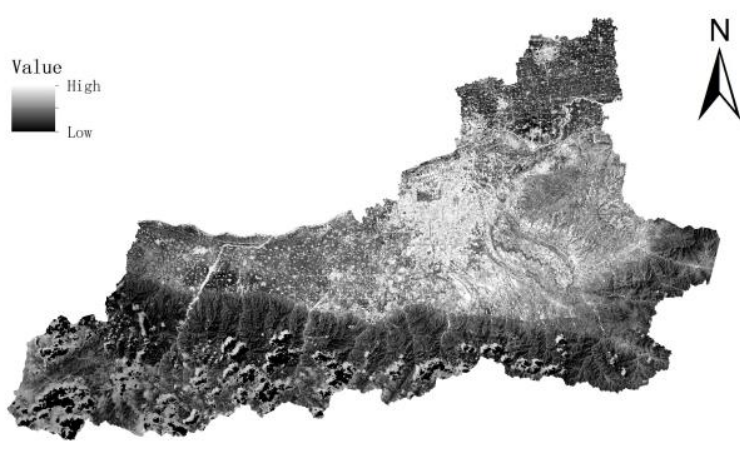

Landsat8 ISA 20140815

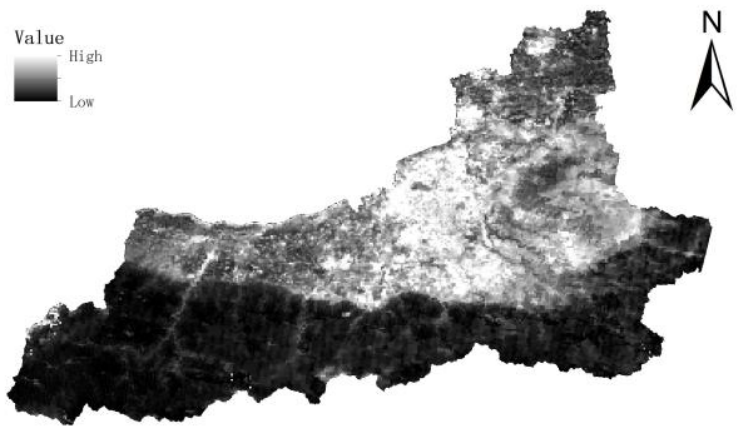

MODIS ISA 20140815

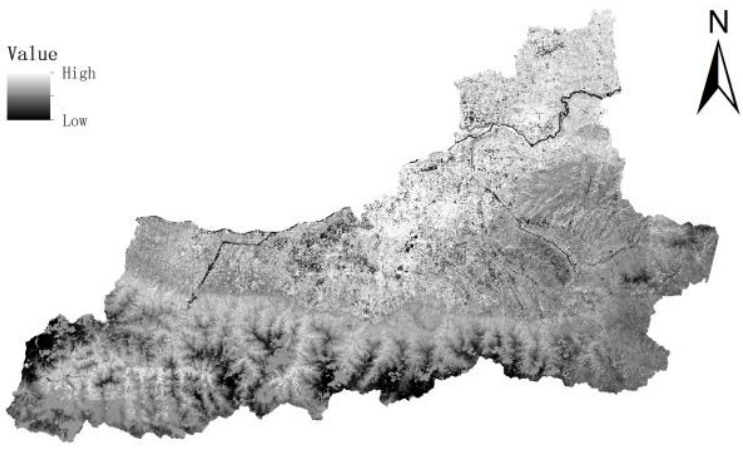

ESTARFM ISA 20140815

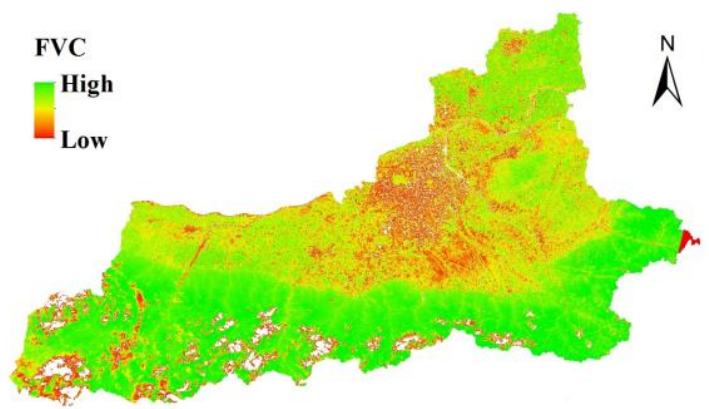

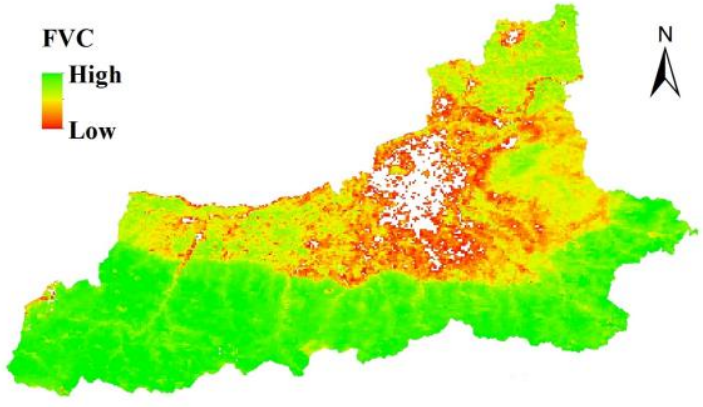

MODIS FVC 20140815

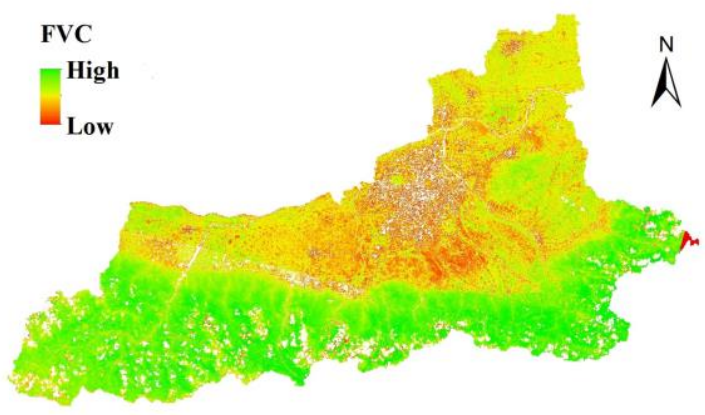

ESTARFM FVC 20140815

Figure 5. The distribution of impermeable layer at study area

\subsection{Quantitative analysis}

ESTARFM impermeable layer data of high temporal and spatial resolution is the effective addition of the spatial information of Landsat8 impermeable layer on MODIS impermeable layer time series data, so it can correctly reflect the data of Landsat8 impermeable layer The spatial information is an important criterion to test the effectiveness of ESTARFM impermeable layer data. The result is shown in figure6.

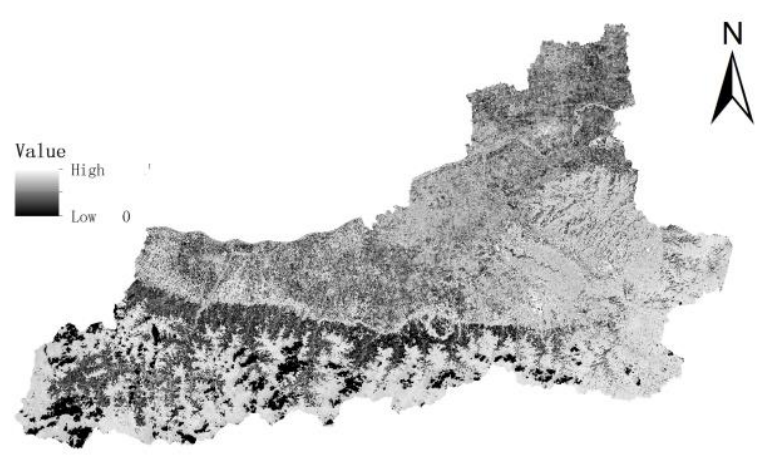

ISA Difference 20140815

Landsat8 FVC 20140815 


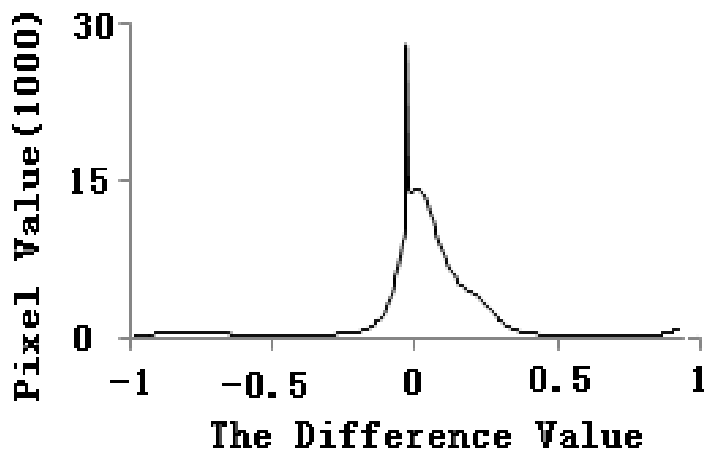

Difference Value of ISA 20140815

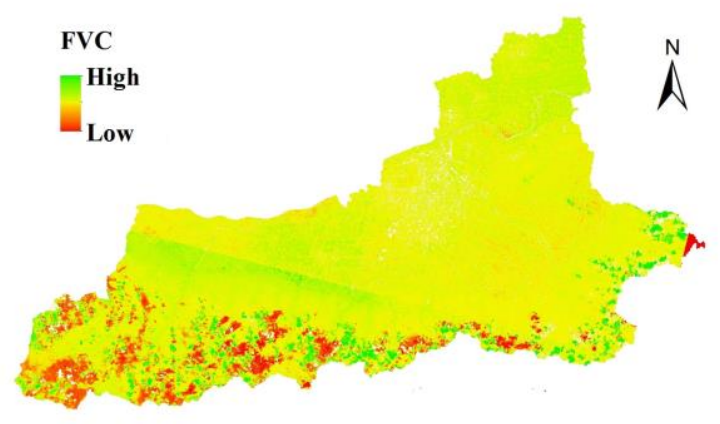

FVC Difference 20140815

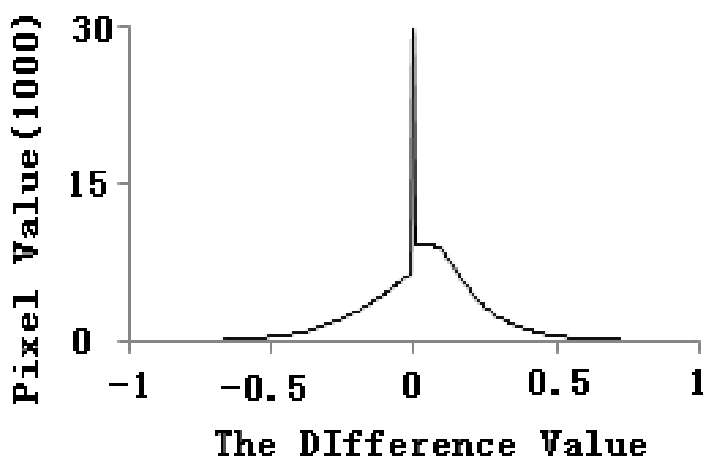

Difference Value of FVC 20140815

Figure 6. The difference between Landsat8 \&ESTARFM

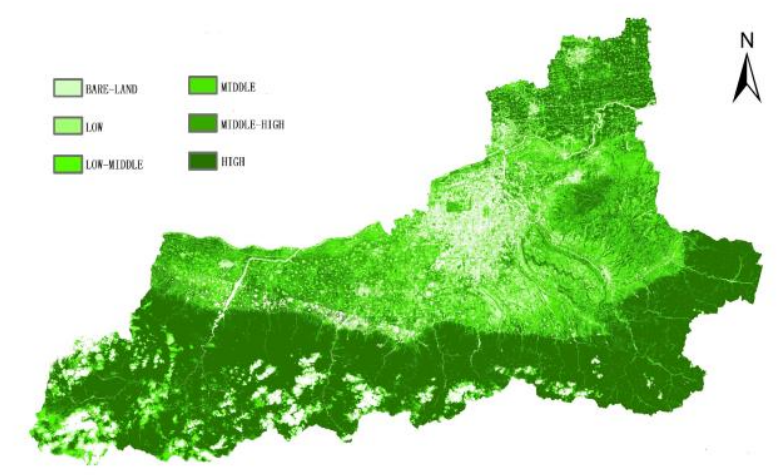

Figure 7. Spatial Distribution of Vegetation Coverage in Xi'an 4.3 Impervious surface and spatial distribution of
vegetation cover characteristics

4.3.1 Spatial Features Analysis: Vegetation coverage in $\mathrm{Xi}$ 'an is a good description of the distribution of vegetation. This section uses the spatial and temporal characteristics of the vegetation coverage data of Xi'an on August 15, 2014 as shown in the figure 7 to analyze the spatial characteristics. The average vegetation coverage in Xi'an was $62.0 \%$ on August 15, 2014. Vegetation coverage was divided into 6 grades according to the classification code of vegetation coverage, and the grading standards were $0-15 \%$ (bare land) and $15-30 \% \%$ of low coverage, $35 \% \sim 45 \%$ of low-middle coverage, $45 \% \sim 60 \%$ of middle coverage, $60 \% \sim 75 \%$ of middle-high coverage, $75 \% \sim$ $100 \%$ of high coverage, There is a more obvious spatial distribution of vegetation coverage in Xi'an. Among them, the vigorous growth of vegetation around the urban area of Xi'an, high coverage and high coverage. The result is shown in figure7.Urban centre vegetation coverage is low. Xi'an urban area has obvious differences in the aspects of industrial location and urban functions, and the difference in the degree and degree of urbanization determines the different characteristics of the surface coverage. The average area of the watertight surface area was between $39.1 \%$ and $50.1 \%$. The city centre has a large degree of impermeability to water and a low degree of impermeability in the suburbs. It can be seen that the spatial distribution of vegetation coverage and impermeable layer in $\mathrm{Xi}^{\prime}$ an is closely related to the topography and human activities. Human beings live in plains with good environment. Affected by their frequent production activities, the vegetation coverage has declined and the impervious cover Area increases; with the city center continues to move closer, the impact of human factors gradually increased, interference with vegetation also will increase, so the coverage level is reduced.

4.3.2 Correlation Analysis of ISA and FVC: Vegetation cover change is an important indicator of regional ecological changes. After the original image is decomposed by linear spectral decomposition, the vegetation terminal component can be used to characterize vegetation abundance. Therefore, the relationship between impervious surface and vegetation can be studied by studying the correlation between ISA and vegetation terminal components. In this paper, the spatial autocorrelation analysis of Moran-I coefficients for continuous variables is chosen to quantitatively measure the spatial dependence of the land cover features on the Moran I coefficients of impervious surfaces and vegetation cover of 0.950 and 0.972 , respectively. The spatial coverage of land cover has the same trend with the neighborhood value. The positive correlation of vegetation coverage is stronger on the impermeable surface, indicating that the vegetation coverage is more spatial congregation.

\section{SUMMARY}

Based on Landsat8 (30m, 16day) data and MODIS (500m, 8day) data for Xi'an in 2014, Landsat8 data were radiated and calibrated, and atmospheric correction and inlay cropping of two-track images in the study area were carried out. The MODIS data were transformed, stitched, resampled. Taking the preprocessed Landsat 8 and MODIS images as the data source, the vegetation coverage data of the two sensor images are obtained according to the pixel dichotomy. Then, ESTARFM model was used to combine the temporal advantage of MODIS data with the spatial advantage of Landsat8 data to generate 8day and $30-\mathrm{m}$ resolution vegetation coverage data and impervious data in the study area. Taking August 15 as an 
example, the spatial characteristics of vegetation coverage in Xi'an were analyzed. The results showed that the vegetation area in the study area was dominated by high vegetation coverage, accounting for $42.9 \%$ of the study area.

1. Based on the classification results, there is still a small amount of bare soil which is misjudged as impervious surface. Some of the bare soil is in fact part of the urban area ready to be built as a flat bare land. Due to the nature of its construction, it is also a watertight surface, so this part of confusion can be ignored and relevant data should be updated

2. ESTARFM FVC and impermeable surface with high accuracy, and can characterize the composition of the surface covered by the biological components;

3. ESTARFM FVC is consistent with Landsat8 FVC in the same period, and the overall prediction accuracy is high;

4. The average water-impermeable surface proportion and spatial configuration of each district are different. Due to the influence of natural conditions and urbanization mode, the urban landscape in $\mathrm{Xi}^{\prime}$ an, which is characterized by typical spontaneous urbanization, is fragmented and has less spatial dependence .

\section{REFERENCES}

Chester L. Arnold Jr, C. James Gibbons.1996. Impervious Surface Coverage: The Emergence of a Key Environmental Indicator. Journal of the American Planning Association, 62(2), pp. 243-258.

Chong Liu, Zhenfeng Shao, Min Chen, et al.2013. MNDISI: a multi-source composition index for impervious surface area estimation at the individual city scale. Remote Sensing Letters, 4(8), pp.803-812.

Hu X F, Weng Q H.2009. Estimating impervious surfaces from medium spatial resolution imagery using the self-organizing map and multi-layer perceptron neural networks. Remote Sensing of Environment, 113(10), pp. 2089-2102.

Gitelson A A, Kaufman Y J, Stark R, et al.2002.Novel algorithms for remote estimation of vegetation fraction. Remote sensing of Environment, 80(1),pp. 76-87.

Goetz S J, Wright R K, Smith A J, et al.2003.IKONOS imagery for resource management: Tree cover, impervious surfaces, and riparian buffer analyses in the mid-Atlantic region. Remote Sensing of Environment, 88(1-2),pp.195-208.

Li D R, Luo H, Shao Z F.2016.Review of Impervious Surface Mapping Using Remote Sensing Technology and Its Application. Geomatics and Information Science of Wuhan University, 41(5),pp.569-57

Lu D, Li G, Kuang W, et al.2014. Methods to extract impervious surface areas from satellite images. International Journal of Digital Earth, 7(2),pp.93-112.

Mohapatra R P, Wu C.2010. High Resolution Impervious Surface Estimation. Photogrammetric Engineering \& Remote Sensing, 76(12),pp.1329-1341.

Moran E F. 2010. Land Cover Classification in a Complex Urban-Rural Landscape with Quickbird Imagery. Photogramm Eng Remote Sensing, 76(10).pp.1159-1168.
Price J C. 1994. How unique are spectral signatures. Remote Sensing of Environment, 49(3),pp.181-186.

Ridd M K.1995. Exploring a V-I-S (vegetation-impervious surface-soil) model for urban ecosystem analysis through remote sensing: comparative anatomy for citiesâ. International Journal of Remote Sensing, 16(12),pp.2165-2185.

Wu C, Murray A T.2003. Estimating impervious surface distribution by spectral mixture analysis. Remote Sensing of Environment, 84(4),pp.493-505.

Zhang Y, Zhang H, Lin H.2014. Improving the impervious surface estimation with combined use of optical and SAR remote sensing images. Remote Sensing of Environment, 141(2),pp.155-167.

Zhu X, Chen J, Gao F, et al.2010. An enhanced spatial and temporal adaptive reflectance fusion model for complex heterogeneous regions. Remote Sensing of Environment, 114(11),pp. 2610-2623. 